\title{
FELICIDAD, SÍNDROME DE BURNOUT Y ESTILOS DE AFRONTAMIENTO EN UNA EMPRESA PRIVADA
} Happiness burnout and coping styles in a private company

\author{
Walter L. Arias*, Alejandra Masías** y Oscar Justo**
}

\begin{abstract}
Resumen
En el presente trabajo se analizan las relaciones entre la felicidad, el síndrome de burnout y los estilos de afrontamiento en 74 trabajadores (44 varones y 30 mujeres) de una empresa privada de Arequipa. Para ello se utilizó la Escala de Felicidad de Lima, el Inventario de Burnout de Maslach y el Cuestionario de Conductas de Afrontamiento ante situaciones de Estrés. Los resultados sugieren que existen relaciones significativas $(p<0.001, p<0.005)$ entre el síndrome de burnout y la felicidad, y el síndrome de burnout y el afrontamiento, mas no entre la felicidad y los estilos de afrontamiento. Además, en los varones de mayor edad hay niveles menores de agotamiento emocional y despersonalización. Mientras que el grado de instrucción se relacionó negativamente en las mujeres, con la baja realización personal. Los estilos de afrontamiento son distintivos de cada género: mientras los varones prefieren un estilo de afrontamiento activo, en las mujeres predominan los estilos evitativo y pasivo, de modo que entre las mujeres existe una relación negativa $(r=-, 372)$ entre la alegría de vivir y el estilo activo de afrontamiento, y entre los varones, el estilo pasivo se relaciona positivamente con la despersonalización y el síndrome de burnout.
\end{abstract}

Palabras Clave: Felicidad, síndrome de burnout, afrontamiento, psicología laboral.

\begin{abstract}
In this paper the relationship between happiness, burnout and coping styles in 74 workers (44 men and 30 women) from a private company Arequipa are analyzed. This Happiness Scale Lima was used, the Maslach Burnout Inventory and Coping Behaviors Questionnaire in situations of stress. The results suggest that there are significant relationships $(p<0.001, p<0.005)$ between burnout and happiness, and burnout and coping, but not between happiness and coping styles. Moreover, in older men's lower levels of emotional exhaustion and depersonalization. While the level of education was negatively associated in women with low personal accomplishment. Coping styles are distinctive from each genre: while men prefer active coping style in women predominate and passive avoidant styles, so that among women there is a negative relationship ( $r$ $=-, 372$ ) between joy live and active coping style, and among men, the passive style is positively related to depersonalization and burnout.
\end{abstract}

Keywords: Happiness, burnout, coping, occupational psychology.

* Docente investigador de la Universidad Católica San Pablo de Arequipa. walterlizandro@hotmail.com

** Estudiante del programa de Psicología de la Universidad Católica San Pablo. 


\section{INTRODUCCIÓN}

Las sociedades están cambiando y con ellas, cambian también las organizaciones (Salanova, Martínez \& Llorens, 2005). Priman, hoy en día, los modelos flexibles de trabajo, que brindan mayor importancia al capital humano, intelectual y psicológico. En ese sentido, se valoran cada vez más las capacidades emocionales y los valores que favorecen las relaciones interpersonales en el contexto laboral (Goleman, 2010). Se brinda mayor autonomía a los trabajadores, las estructuras organizacionales tienden a ser horizontales, se alienta la participación de los empleados en la toma de decisiones y el involucramiento con el trabajo, etc.

Sin embargo, estos principios que orientan el funcionamiento de las organizaciones del siglo XXI, están siendo lentamente introducidos en las empresas latinoamericanas. De hecho, en los países latinoamericanos los trabajadores se encuentran más distanciados de los puestos jerárquicos más elevados, y muestran mayor aceptación hacia las formas de liderazgo autocrático con desigual distribución de poder. Los trabajadores latinos son también más colectivistas y proclives a no cumplir las normas internas, tampoco se preocupan mucho por la seguridad y desarrollan formas de afrontamiento centradas en las relaciones humanas (Izquierdo \& Alonso, 2010). A esto se suma que los factores de riesgo psicosocial sean relegados en las empresas de los países en vías de desarrollo, ya que se presta mayor atención a los riesgos físicos, biológicos y ambientales (Gómez, 2010). La situación laboral es también más inestable, por lo que el desempleo constituye un factor que afecta la identidad personal de los trabajadores y se asocia con emociones negativas y el consumo de sustancias psicoactivas, Ardila (1991).

En consecuencia, las condiciones de trabajo se combinan condicionando el caldo de cultivo ideal para la aparición y el mantenimiento de estrés laboral, con las correspondientes emociones negativas que éste acarrea. El estrés en el trabajo, puede definirse, como la respuesta física y emocional nociva que ocurre cuando los requerimientos de trabajo no son compatibles con las capacidades, los recursos o las necesidades de los trabajadores (García, 2003). Los estudios sobre el estrés laboral, han cobrado vital importancia en los últimos años, debido a que el estrés merma la salud de los trabajadores y su disposición para trabajar (Schultz, 1998) y se asocia positivamente con la accidentabilidad en el trabajo (López, \& Osca, 2010). Al respecto, cabe recordar que la capacidad de trabajar es un parámetro de salud psicofísica fundamental (Alonso, 2009), aunque tenga un significado muy particular para cada persona. En una investigación, que indagaba sobre los significados que se le atribuye al trabajo, Pérez (1996) concluyó que los trabajadores que consideran el trabajo como "libertad" y "creación" están implicados, tanto con el goce y el disfrute de las tareas que desempeñan, como con la potencia, la realización y la felicidad, en mayor medida, que quienes consideran el trabajo como una necesidad o un medio para obtener dinero. De hecho, el trabajo aumenta la satisfacción respecto de la vida y contribuye a la felicidad de las personas (Niven, 2009).

En general, la principal causa de estrés en los trabajadores, es un ambiente de trabajo tenso, lo que ocurre cuando el modelo laboral es muy autoritario y estos trabajadores no tienen oportunidad de intervenir en la toma de decisiones. Entre los factores específicos que se relacionan con el estrés laboral se tiene: los turnos de trabajo seguidos y la sobrecarga laboral, la inestabilidad del puesto, la antigüedad profesional, la incorporación de nuevas tecnologías, el clima organizacional negativo, la falta de retroalimentación, la escasa oportunidad de control, el bajo salario, las relaciones interpersonales conflictivas y las estrategias empresariales parcializadas que atentan contra la equidad (Apiquian, 2007). Cuando estas condiciones de trabajo perduran en el tiempo, el estrés se hace crónico, y si el trabajo implica un constante contacto con otras personas en situación de dependencia, se hacen patentes los síntomas propios del síndrome de burnout o el "estar quemado por el trabajo". De este modo, el síndrome de burnout puede definirse como la sensación de agotamiento, decepción y pérdida de interés por la actividad laboral, que surge, especialmente, en aquellas personas que se dedican a profesiones de servicio, como consecuencia del contacto diario con su trabajo (Borda, Navarro, Aun, Berdejo, Racedo \& Ruíz, 2007).

Precisamente, las profesiones más vulnerables son las de enseñanza, seguridad y cuidado de la salud 
física y mental (Gil-Monte \& Peiró, 1999a). Sólo recientemente, se está estudiando el síndrome de burnout en personal administrativo, en particular, el tipo de puestos que implican la atención de clientes. En nuestro país, los trabajadores de empresas públicas tienen mayor riesgo de padecer este síndrome que los que laboran en empresas privadas, presentando mayores niveles de estrés y de insatisfacción (Fernández, 2010). Aunque los síntomas principales abarcan la triada: agotamiento emocional, despersonalización y baja realización personal; también se observa la pérdida de autoestima profesional, la falta de satisfacción en el trabajo, los conflictos interpersonales y las alteraciones somáticas (Guillén, Guil \& Mestre, 2000).

En una investigación realizada por Boada, De Diego y Agulló (2004), en 137 trabajadores españoles, se encontró que la proliferación de tareas y la utilización excesiva de destrezas que demanda el cargo, acrecientan las disfunciones respiratorias y del sueño, así como la sensación de estar agotado. Asimismo, la independencia en el trabajo mejora la realización personal, mientras que la carencia de autonomía incrementa las jaquecas. La significatividad percibida del trabajo y el conocimiento de los resultados del trabajo se correlacionaron positivamente con el síndrome de burnout y las dimensiones de agotamiento emocional y despersonalización. Es decir, que como ha señalado García (2003), las condiciones de trabajo provocan alteraciones en la salud, tanto física como mental de los trabajadores.

Ahora bien, distintos autores proponen reconceptualizar las experiencias traumáticas desde un modelo salutogénico, que basado en métodos positivos de prevención, tenga en consideración la habilidad natural de los individuos de afrontar, resistir e incluso de aprender y crecer en las situaciones más adversas (Lamas, 2004). Así por ejemplo, la resiliencia se define como la capacidad para superar la adversidad a través de la adaptación exitosa (Ospina, 2007), el hardiness hace referencia a la personalidad resistente que permite enfrentar las adversidades. Este constructo planteado por Kobasa y Maddi en 1972, abarca componentes como compromiso, control y reto. La diferencia entre los individuos resistentes y los resilientes, es que mientras los primeros se sobreponen a la adversidad, lo cual implica un descenso temporal de sus recursos emocionales, los segundos se mantienen en niveles normales de funcionamiento (Lamas, 2004). Otro constructo relacionado es el de crecimiento posttraumático, que alude la habilidad para sobreponerse a las experiencias negativas y cambiar positivamente.

El denominador común de estas habilidades son las emociones positivas, que ejercen un efecto protector frente a la depresión, la ansiedad y el estrés; impulsando el ajuste funcional de las personas (Fredrickson, 2009). Las emociones positivas pueden constituirse entonces, como variables que designan el estilo de afrontamiento de las personas en diversos contextos, incluyendo el laboral. El afrontamiento, es el esfuerzo ,tanto cognitivo como emocional y conductual, que realiza el individuo para hacer frente al estrés (Sandín, 1995). Se han distinguido diversas formas de afrontamiento que responden a variados modelos teóricos. De acuerdo con Labrador (1996), los estilos de afrontamiento más comunes son: el activo, el evitativo y el pasivo. El estilo pasivo, implica que la persona simplemente deja que las cosas pasen, y se ha asociado con mayor malestar psicológico o distrés. El estilo evitativo, se caracteriza porque la persona evita los estresores. Este tipo de respuesta se asocia con altos niveles de ansiedad; mientras que el estilo activo, en el que se confronta el estrés conductual, emocional o cognitivamente, se acompaña de efectos fisiológicos que inducen la cardiopatía coronaria y la hipertensión. Sin embargo, el estilo de afrontamiento activo, puede ser saludable ,si se asume de manera constructiva y relajada (Magnuson, citado por Sandín, 1995), por ejemplo, cuando se trabaja en equipo o se busca apoyo social como modo característico de afrontar el estrés laboral (Apiquian, 2007).

En ese sentido, Roca y Torres (2001) han estudiado el síndrome de burnout y el sentido de coherencia, entendiendo por "sentido de coherencia" la disposición que tiene la persona de utilizar estrategias de afrontamiento como una orientación salutogénica dirigida a la búsqueda de factores promotores de salud y de bienestar. Este concepto, propuesto por Aaron Antonovsky en 1987, abarca tres dimensiones: 1) la comprensión de las condiciones estresantes en el trabajo y su pertinente reestructuración cognitiva, 2) la manejabilidad, hace referencia a la percepción que tiene la persona de sus recursos disponibles para afrontar las 
demandas del ambiente, y 3) la significatividad, que constituye el elemento que moviliza los recursos de afrontamiento del trabajador hacia formas efectivas de reducir el estrés. Los hallazgos de Roca y Torres sugieren que los profesionales de la salud, que cuentan con alto sentido de coherencia, presentan menos niveles de agotamiento emocional y despersonalización. De modo que las características personales del trabajador, juegan un rol importante, como mediadores del estrés laboral y las manifestaciones del síndrome de burnout. De hecho, las características del propio trabajo y del trabajador condicionan su respuesta afectiva. En otro estudio que analiza las relaciones entre satisfacción laboral y síndrome de burnout en trabajadores inmigrantes que ocupan puestos poco cualificados, se encontró que la satisfacción con el ocio genera buen humor, felicidad y satisfacción laboral. Además, la inestabilidad laboral se asoció, significativamente, con la baja realización personal, sobre todo en los varones (Moreno, Ríos, Canto, San Martín \& Perles, 2010).

Todos estos alcances, respecto de los beneficios que reporta el humor, la satisfacción y la felicidad, han sido englobados por el marco epistemológico de la psicología positiva, que propone un nuevo modelo para afrontar el estrés laboral. Este modelo o corriente psicológica aboga por una visión más optimista del hombre y se centra en la vivencia y promoción de emociones como felicidad, risa, buen humor, esperanza, gratitud, etc. (Alarcón, 2009). Dado que históricamente, la psicología se ha centrado en el estudio de los afectos negativos, la psicología positiva amplía su foco de atención más allá del sufrimiento y su consecuente alivio, pero no pretende trasladar el foco de investigación de lo negativo a lo positivo, sino abordar el estudio del ser humano desde una perspectiva integral (Greco, 2010). Es decir que, como dice Alarcón (2011), "la psicología positiva es un complemento de la psicología negativa, no su sustituto" (p. 198).

Por tanto, no se debe dejar de investigar las causas y las consecuencias de los afectos negativos, sino que se debe complementar estos estudios con otros, que permitan profundizar en el conocimiento de los beneficios de las emociones positivas y sus aplicaciones en el trabajo. En ese sentido, Kim Cameron ha sido pionero en realizar estudios sobre las aplicaciones de la psicología positiva al contexto laboral (Page, Govindji, Carter \& Linley, 2008). Asimismo, Luthans (citado por Salanova, Martínez \& Llorens, 2005) señaló la importancia de una aproximación proactiva en la investigación de la psicología organizacional, a la que llamó Conducta Organizacional Positiva. Este enfoque implica el estudio y la aplicación de recursos y competencias humanas, que pueden ser medidas, desarrolladas y gestionadas con el fin de mejorar el desempeño en las organizaciones. Es decir, se centra en las fortalezas de los trabajadores y potencia sus conocimientos, sus emociones, sus habilidades sociales y sus competencias profesionales; para orientarlos positiva y eficazmente según los objetivos organizacionales de cada empresa.

Podemos decir entonces que, aunque los modelos conductuales de evaluación y valoración del medio organizacional, han sido útiles e importantes (López, 1993), están siendo reemplazados por enfoques más positivos, en los que se privilegia la vivencia de emociones que conllevan al engagement y el flow laboral. Por engagement se entiende un estado afectivo positivo de plenitud que se caracteriza por el vigor, la dedicación, y la absorción o concentración en el trabajo. El flow tiene que ver con el disfrute que ocurre cuando una persona está motivada y capacitada para realizar una actividad porque se siente desafiada, de modo que la actividad laboral es satisfactoria en sí misma (Salanova, Martínez \& Llorens, 2005). En ambos casos se promueve el humor y los sentimientos de involucramiento, que devienen en la alta motivación hacia el trabajo. Los beneficios del humor, en un contexto empresarial, se han probado eficazmente en las ventas, y se sabe que proporcionan tranquilidad mental en el trabajo (Jáuregui, 2008). Asimismo, el humor evita caer en la desesperanza (Zavala, 2009) y se asocia con una visión optimista del entorno laboral y la satisfacción en el trabajo.

Es decir que, como dice Seligman (2006), en el trabajo se está pasando rápidamente de una economía monetaria a una economía centrada en la satisfacción con la vida (Seligman, 2006), en el que la felicidad del trabajador ocupa un lugar central. Pero, hablar de psicología positiva en el trabajo, no es sólo alentar el buen humor entre los empleados, ya que tiene más implicancias, como establecer políticas 
organizacionales y laborales más saludables, realizar investigación constante y diseñar programas basados en la felicidad (Villanueva, 2004). En nuestro país, David Fischman ha aplicado en un banco de Lima, un programa denominado "Alta rentabilidad de la felicidad", que abarca el entrenamiento en una diversidad de estrategias que se focalizan en las emociones positivas. Sus resultados, indican que los empleados que participaron del taller de la alta rentabilidad de la felicidad reportaron en promedio ser más felices que los que trabajaron el taller de negociación del grupo control (Fischman, 2011).

La felicidad se ha definido como un estado de bienestar subjetivo o como sentir satisfacción con la vida, aunque Alarcón (2006) señala que ambos conceptos, el de felicidad y el de satisfacción, se refieren a cosas diferentes. Abordar el problema del trabajo, desde una perspectiva de la felicidad, implica proporcionar elementos necesarios para que los trabajadores accedan a zonas de flujo positivo, y escenarios laborales de acción positiva (Waugh \& Fredrickson, 2006), como en el caso del estudio de Fischman. Lamentablemente, son todavía pocos los estudios sobre la felicidad en el trabajo. Mucho menos aún, son los estudios que intentan encontrar relaciones entre los afectos positivos y negativos, como expresión de un enfoque integral que apunta a la prevención de riesgos psicosociales, ya que la comprensión de los riesgos laborales no puede ser asociada linealmente a la presencia de un factor potencialmente nocivo para la salud (Hernández, Almirall \& Franco, 2005), sino que se deben analizar estas variables en sus relaciones multidireccionales. En ese sentido, el presente estudio busca hallar las relaciones entre la felicidad, el síndrome de burnout y los estilos de afrontamiento.

\section{MÉTODO}

\section{Muestra}

La muestra está conformada por 74 trabajadores de una empresa privada de Arequipa (44 varones y 30 mujeres), que se dedica a la prestación de servicios. La cantidad de participantes, se determinó mediante el muestreo simple de una población de 580 empleados, con un nivel de confianza del 95\%. Para la selección de la muestra se utilizaron métodos probabilísticos, mediante el uso de números aleatorios, hasta conformar la cantidad de participantes requeridos a fin de trabajar con una muestra representativa.

\section{Instrumentos}

Como instrumentos de investigación se utilizaron, una Ficha de datos personales, la Escala de Felicidad de Lima, el Inventario de Burnout de Maslach y el Cuestionario de Conductas de Afrontamiento en situaciones de Estrés. La ficha de datos personales permitió recoger datos tales como edad, sexo, tiempo de servicio, área en que trabaja, grado de instrucción, estado civil y número de hijos.

La Escala de Felicidad de Lima (EFL) fue elaborada y validada por Reynaldo Alarcón (2006) en una población heterogénea de habitantes de Lima con un índice de confiabilidad de 0.912 . La escala consta de 27 preguntas con cinco alternativas de respuesta tipo Likert: Totalmente de acuerdo, Acuerdo, Ni acuerdo ni desacuerdo, Desacuerdo y Totalmente en desacuerdo. Alarcón ha determinado además mediante un análisis factorial, 4 factores de la felicidad: El factor 1 se refiere al Sentido positivo de la vida, el factor 2 a la Satisfacción con la vida, el factor 3 a la Realización personal y el factor 4 se refiere a la Alegría de vivir. Asimismo, se obtuvieron niveles aceptables de consistencia interna mediante el coeficiente Alfa de Cronbach, para cada uno de los 4 factores de la escala de felicidad (factor $1 \alpha=.864$, factor $2 \alpha=.706$, factor $3 \alpha=.780$, factor $4 \alpha=.766$ ), así como para la escala global $(\alpha=.863)$.

El Inventario de Burnout de Maslach (IBM) forma genérica, que fue validada por Gil-Monte y Peiró(1999), en una muestra multiocupacional hispanohablante, con un índice de confiabilidad de 0.79. Este inventario, consta de 22 ítems de carácter autoafirmativo, con una escala de frecuencia de 7 grados que va de 0 (nunca) a 6 (todos los días), tipo Likert. El instrumento, se compone de tres subescalas, que corresponden a las tres dimensiones del síndrome de burnout: agotamiento emocional (9 ítems), despersonalización (5 ítems) y baja realización personal (8 ítems). Se hallaron niveles de consistencia interna óptimos, mediante el coeficiente Alfa de Cronbach: burnout $\alpha=.721$, agotamiento emocional $\alpha=.761$, despersonalización $\alpha=.606$, y baja realización personal $\alpha=.652$. 
El Cuestionario de Conductas de Afrontamiento en situaciones de Estrés de Magnuson validado por José Francisco Labrador (1996) en España. El cuestionario, tiene 24 ítems con cinco alternativas de respuesta tipo Likert: Nunca lo hago, casi nunca lo hago, a veces lo hago, casi siempre lo hago y siempre lo hago. El cuestionario, permite identificar tres estilos de afrontamiento: activo, pasivo y evitativo con 8 ítems cada uno. Se obtuvo un coeficiente Alfa de Cronbach $\alpha=.690$ de consistencia interna.

\section{Procedimientos}

Se procedió, en primer lugar, a solicitar los permisos correspondientes ante las autoridades de la empresa, los mismos que nos fueron concedidos, siempre y cuando se mantuviera el anonimato de los datos de los trabajadores así como de la institución. Los datos, fueron recolectados en los momentos de descanso de los trabajadores, durante los meses de noviembre y diciembre del 2011, y se procesaron mediante el paquete estadístico SPSS 16.0, para estudios sociales licenciado por Microsoft.

\section{RESULTADOS}

Presentaremos los datos según el sexo de los sujetos de la muestra, ya que no fue posible equiparar el número de trabajadores en función de esta variable. Asimismo, aunque se les pidió a los trabajadores que llenen todos los datos en la ficha anónima de datos personales, muchos de ellos se negaron a escribir el área en que trabajan porque refirieron que podrían ser fácilmente identificados. De modo, que se procesó la información, prescindiendo de este dato. Para el procesamiento de los datos, se han utilizado estadísticos descriptivos y de frecuencias, además de pruebas paramétricas de correlación (Coeficiente de Pearson) para las variables cuantitativas y pruebas no paramétricas (Prueba Tau-b de Kendall) para las variables cualitativas, mediante el programa SPSS 16.0.

\section{Análisis de datos de trabajadores de sexo masculino}

La muestra de trabajadores varones tiene una edad promedio de 35,90 años y una desviación estándar de $10,195 \pm$. En cuanto al grado de instrucción el 6,8\% son estudiantes universitarios al igual que los bachilleres, $2,3 \%$ son técnicos, $65,9 \%$ son profesionales titulados y $13,6 \%$ tienen grado de magíster y $4,5 \%$ tienen grado de doctor. Además, el $25 \%$ tiene un año de tiempo de servicio, mientras que $38,7 \%$ lleva trabajando de dos a cinco años, y el 36,3\% trabaja más de cinco años en la empresa. La media para el tiempo de servicio es de 5,318 años, con una desviación estándar de 4,806士. Con respecto al estado civil, $36,4 \%$ son solteros, $59,1 \%$ son casados, $2,3 \%$ son divorciados y $2,3 \%$ son convivientes. El $50 \%$ no tiene hijos, mientras que $9,1 \%$ tiene un hijo, 31,8\% tiene dos hijos y el 9,1\% tiene entre 3 y 4 hijos.

Tabla 1. Descriptivos de la felicidad, burnout y estilos de afrontamiento en los trabajadores varones

\begin{tabular}{lrccccc}
\hline Variables y dimensiones & Media & Desviación estándar & Mínimo & Máximo & Rango & Varianza \\
\hline Felicidad & 111,931 & 12,965 & 76 & 135 & 59 & 168,11 \\
Sentido positivo & 49,000 & 6,239 & 32 & 59 & 27 & 38,930 \\
Satisfacción con la vida & 23,113 & 3,654 & 14 & 29 & 15 & 13,359 \\
Realización personal & 22,181 & 4,053 & 12 & 30 & 18 & 16,431 \\
Alegría de vivir & 17,568 & 1,619 & 14 & 20 & 6 & 2,632 \\
Síndrome de burnout & 57,636 & 14,049 & 16 & 92 & 76 & 197,400 \\
Agotamiento emocional & 14,045 & 8,123 & 0 & 38 & 38 & 65,998 \\
Despersonalización & 4,727 & 4,520 & 0 & 19 & 19 & 20,436 \\
Baja realización personal & 38,613 & 8,575 & 7 & 50 & 43 & 73,545 \\
Afrontamiento activo & 15,363 & 4,735 & 7 & 26 & 19 & 22,423 \\
Afrontamiento evitativo & 12,931 & 5,132 & 4 & 26 & 22 & 26,344 \\
Afrontamiento pasivo & 11,545 & 4,697 & 3 & 27 & 24 & 22,068 \\
\hline
\end{tabular}


Los descriptores de cada una de las variables y sus dimensiones se muestran en la Tabla 1. Como puede apreciarse, las medidas de felicidad y sus dimensiones son elevadas. Con respecto a los porcentajes, tenemos que $79,5 \%$ presenta leves niveles de agotamiento emocional, 13,6\% tiene niveles moderados y 6,8\% severos. El $61,4 \%$ de trabajadores tiene un nivel leve de despersonalización, 31,8\% moderado y $6,8 \%$ severo. El 54,4\% tiene un leve nivel de baja realización personal, 27,3\% moderado y 18,2\% severo. Asimismo, en cuanto a los estilos de afrontamiento, el 54,5\% tiene un estilo activo, mientras que el $27,3 \%$ tiene un estilo evitativo y el $18,2 \%$ tiene un estilo pasivo.

A continuación, se presentan las correlaciones obtenidas entre las diversas variables y sus dimensiones. Es decir, entre las variables sociolaborales, la felicidad y sus cuatro factores, el síndrome de burnout con sus tres dimensiones, y los estilos de Afrontamiento.

Tabla 2. Correlaciones entre datos personales, felicidad, burnout y estilos de afrontamiento en varones

\begin{tabular}{lccccc}
\hline Variables y dimensiones & Edad & Tiempo de servicio & Grado Instrucción & Estado civil & Número de hijos \\
\hline Felicidad &, 150 &, 093 &,- 089 &,- 082 &, 190 \\
Sentido positivo &, 033 & -065 &,- 117 &,- 053 &, 084 \\
Satisfacción &, 096 &,- 048 &, 029 &,- 161 &, 126 \\
Realización &, 133 &, 091 &,- 001 &,- 137 &, 205 \\
Alegría de vivir &,- 170 &,- 084 &,- 145 &,- 005 &,- 077 \\
Sínd. de burnout &,- 284 &,- 140 &, 125 &,- 032 &,- 044 \\
Agotamiento &,$- 453 *$ &,- 177 &, 118 &,- 073 &,- 177 \\
Despersonalización &,$- 414^{*}$ &,- 098 &, 143 &, 017 &,- 005 \\
Baja realización &,- 003 &, 222 &, 025 &,- 009 &, 083 \\
Estilo activo &,- 162 &,- 110 &, 144 &,- 238 &,$- 335 * *$ \\
Estilo evitativo &, 071 &, 156 &, 020 &, 052 &, 033 \\
Estilo pasivo & $-0,81$ &, 070 &,- 044 &, 169 &, $299 * *$ \\
\hline
\end{tabular}

$* \mathrm{p}<.001, * * \mathrm{p}<.005$

Observamos en la Tabla 2 que se encontraron correlaciones negativas moderadas entre la edad y el agotamiento emocional, así como entre la edad y la despersonalización, con un nivel de significancia $\mathrm{p}<$ .001. Ello nos sugiere, que los trabajadores varones de mayor edad, tienen menos agotamiento emocional y se sienten menos despersonalizados. Por otro lado, el número de hijos se relaciona negativamente con el estilo activo de afrontamiento, es decir que a mayor número de hijos el estilo de afrontamiento tiende a ser menos activo. Asimismo, entre el número de hijos y el estilo de afrontamiento pasivo se encontraron relaciones significativas $(p<.005)$, lo que corrobora que a mayor número de hijos el estilo de afrontamiento tiende a ser más pasivo. 
Tabla 3. Correlaciones entre felicidad y burnout y estilos de afrontamiento en trabajadores varones

\begin{tabular}{lrrrrrrrrrrr}
\hline & Felic & Posit & \multicolumn{1}{c}{ Satis } & Real & Aleg & Burn & Agot & Desp & B real & Activ & Evit \\
\hline Posit &, $828^{* *}$ & & & & & & & & & & \\
Satis &, $681^{* *}$ &, $418^{* *}$ & & & & & & & & & \\
Real &, $794^{* *}$ &, $507^{* *}$ &, $593^{* *}$ & & & & & & & & \\
Aleg &, $540^{* *}$ &, $562^{* *}$ &, $425^{* *}$ &, $395^{* *}$ & & & & & & & \\
Burn &, 100 &, 052 &, 060 &,- 019 &, 155 & & & & & & \\
Agot &,- 050 &,- 024 &,- 160 &,- 127 &, 017 &, $599^{* *}$ & & & & & \\
Desp &,- 276 &,- 213 &,- 236 &,$- 341^{*}$ &,$- 380^{*}$ &, $327^{*}$ &, $465^{* *}$ & & & & \\
B real &, 290 &, 253 &, 192 &,- 342 & 0,287 &, $602^{* *}$ &, 056 &,- 018 & & & \\
Activ &, 071 &, 085 &, 093 &, 106 &, 091 &, 143 &, 000 &,- 012 &, 128 & & \\
Evit &, 088 &, 125 &,- 104 &,- 039 &, 036 &, 095 &, 244 &, 230 &,- 018 &,$- 336^{*}$ & \\
Pasiv &,- 009 &,- 027 &,- 071 &,- 055 &,- 106 &, $378^{*}$ &, $386^{* *}$ &, $354^{*}$ &, 224 &,$- 332^{*}$ &, $402^{* *}$ \\
\hline
\end{tabular}

$* \mathrm{p}<.000, * * \mathrm{p}<.005$

Finalmente, con respecto a las correlaciones entre las variables de estudio, tenemos que todos los factores de la escala de felicidad correlacionan positiva y significativamente entre sí. Algo similar ocurre con las dimensiones del inventario de burnout, ya que la puntuación total de burnout correlaciona positiva y significativamente con las tres dimensiones que componen el síndrome; mientras que el estilo activo de afrontamiento correlaciona negativa y significativamente con el estilo evitativo y el estilo pasivo, en tanto que el estilo evitativo y el pasivo correlacionan positivamente. Otras correlaciones significativas se encontraron entre la alegría de vivir y la despersonalización $(\mathrm{r}=-, 380)$, el agotamiento emocional y el estilo pasivo $(\mathrm{r}=386)$, la despersonalización y el estilo pasivo $(\mathrm{r}=, 354)$, y la realización personal y la despersonalización $(\mathrm{r}=-, 341)$ (ver Tabla 3).

\section{Análisis de datos de trabajadores de sexo} femenino

La muestra de trabajadores mujeres tiene una edad promedio de 34,93 años y una desviación estándar de

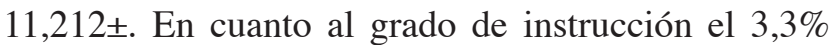
son estudiantes universitarias, $13,3 \%$ tienen una carrera técnica, $76,6 \%$ son profesionales tituladas y
$6,7 \%$ tienen grado de magíster, ninguna trabajadora tiene grado de doctor. En cuanto al tiempo que llevan trabajando en la empresa, el 36,7\% tiene un año de tiempo de servicio, el 40,1\% lleva trabajando de dos a cinco años y el $23,2 \%$ trabaja más de cinco años en la empresa. La media para el tiempo de servicio es de

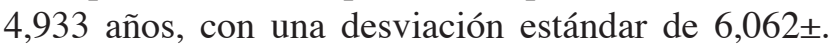
Sobre el estado civil, $50 \%$ son solteras, $46,7 \%$ están casadas y 3,3\% son divorciadas. El $40 \%$ de las mujeres no tiene hijos, el $20 \%$ tiene un hijo, 26,7\% tiene dos hijos y el 13,4\% tiene entre 3 y 4 hijos. 
Tabla 4. Descriptivos de la felicidad, burnout y estilos de afrontamiento en las trabajadoras mujeres

\begin{tabular}{|c|c|c|c|c|c|c|}
\hline Variables y dimensiones & Media & Desviación estándar & Mínimo & Máximo & Rango & Varianza \\
\hline Felicidad & 112,266 & 15,037 & 73 & 135 & 62 & 226,133 \\
\hline Sentido positivo & 47,666 & 9,278 & 16 & 55 & 39 & 86,092 \\
\hline Satisfacción con la vida & 24,266 & 2,933 & 16 & 29 & 13 & 8,961 \\
\hline Realización personal & 22,433 & 4,407 & 12 & 29 & 17 & 19,426 \\
\hline Alegría de vivir & 18,000 & 2,810 & 12 & 18 & 16 & 7,931 \\
\hline Síndrome de burnout & 60,433 & 9,054 & 44 & 83 & 39 & 81,978 \\
\hline Agotamiento emocional & 17,000 & 9,766 & 2 & 33 & 31 & 95,379 \\
\hline Despersonalización & 4,300 & 4,480 & 0 & 15 & 15 & 20,079 \\
\hline Baja realización personal & 40,600 & 7,374 & 22 & 48 & 26 & 54,386 \\
\hline Afrontamiento activo & 13,666 & 5,261 & 0 & 22 & 22 & 27,678 \\
\hline Afrontamiento evitativo & 13,533 & 5,426 & 0 & 24 & 24 & 29,982 \\
\hline Afrontamiento pasivo & 13,733 & 5,475 & 0 & 23 & 23 & 29,444 \\
\hline
\end{tabular}

Los descriptores de cada una de las variables y sus respectivos factores y dimensiones se muestran en la Tabla 4. Como se puede ver, las medidas de felicidad y sus dimensiones son elevadas. En cuanto al síndrome de burnout, el 63,3\% tiene un nivel leve de agotamiento emocional, $13,3 \%$ moderado y $23,3 \%$ severo. El 76,7\% de trabajadoras tiene un nivel leve de despersonalización, $10 \%$ moderado y $13,3 \%$ severo. El $73,3 \%$ tiene un leve nivel de baja realización personal, $10 \%$ moderado y $16,7 \%$ severo. Además, el 36,7\% tiene un estilo activo de afrontamiento, el 30\% tiene un estilo evitativo de afrontamiento y el 33,3\% tiene un estilo pasivo.

Tabla 5. Correlaciones entre datos personales, felicidad, burnout y estilos de afrontamiento en mujeres

\begin{tabular}{lccccc}
\hline Variables y dimensiones & Edad & Tiempo de servicio & Grado de Instrucción & Estado civil & Número de hijos \\
\hline Felicidad &, 060 &, 261 &,- 106 &,- 037 &,- 058 \\
Sentido positivo &, 102 &, 235 &,- 008 &,- 093 &,- 096 \\
Satisfacción & 0,51 &, 125 &, 000 &, 026 &, 087 \\
Realización &,- 048 &, 226 &,- 196 &,- 049 &,- 060 \\
Alegría de vivir &, 080 &, 240 &,- 016 &,- 013 &, 128 \\
Sínd. de burnout &,- 131 &,- 086 &,- 007 &, 057 &,- 029 \\
Agotamiento &, 020 &,- 321 &,- 018 &, 078 &,- 111 \\
Despersonalización &, 097 &,- 138 &,- 008 &, 000 &,- 116 \\
Baja realización &,- 128 &,- 137 &,$- 353^{*}$ &, 114 &,- 359 \\
Estilo activo &, 004 &,- 069 &,$- 378^{*}$ &,- 217 &,- 243 \\
Estilo evitativo &, 102 &, 229 &,- 096 &,- 209 &,- 042 \\
Estilo pasivo &, 122 &, 244 &,- 086 &,- 054 &,- 053 \\
\hline
\end{tabular}

$* \mathrm{p}<.005$ 
En la Tabla 5 se observa que entre el grado de instrucción y la dimensión de baja realización personal del síndrome de burnout, así como entre el grado de instrucción y el estilo activo de afrontamiento, hallamos correlaciones negativas moderadas, con un nivel de significancia $\mathrm{p}<.005$. Es decir que, a mayor grado de instrucción, menor es la baja realización personal y menor es el uso de un estilo de afrontamiento activo.

Para el caso de las trabajadoras de sexo femenino, se han encontrado relaciones significativas entre la mayoría de los factores de la escala de felicidad, a excepción de los factores sentido positivo de la vida y alegría de vivir, y entre satisfacción con la vida y sentido positivo de la vida. En cuanto al síndrome de burnout, las relaciones significativas se dieron entre la felicidad y el agotamiento emocional $(r=-$ ,361), el agotamiento emocional y la satisfacción con la vida $(r=-, 506)$, el agotamiento emocional y la realización personal $(\mathrm{r}=-, 555)$, así como entre la despersonalización y el agotamiento emocional ( $\mathrm{r}=$ ,526), la despersonalización y la realización personal $(r=-, 518)$. Los estilos de afrontamiento, no registran relaciones significativas, salvo entre el estilo activo y la alegría de vivir $(\mathrm{r}=-, 372)$, el estilo pasivo y el sentido positivo de la vida $(\mathrm{r}=, 367)$, el estilo evitativo y la baja realización personal $(\mathrm{r}=-, 441)$, y el estilo evitativo con el estilo pasivo $(r=, 719)$ (ver Tabla 6).

Tabla 6. Correlaciones entre felicidad y burnout y estilos de afrontamiento en trabajadoras mujeres

\begin{tabular}{|c|c|c|c|c|c|c|c|c|c|c|c|}
\hline & Felic & Posit & Satis & Real & Aleg & Burn & Agot & Desp & B real & Activ & Evit \\
\hline Posit &, $856^{*}$ & & & & & & & & & & \\
\hline Satis &, $677^{*}$ & ,268 & & & & & & & & & \\
\hline Real &, $782 *$ &, $438 \dagger$ & ,691* & & & & & & & & \\
\hline Aleg &, $572 * *$ & ,313 &, $581 * *$ &, $453 \dagger$ & & & & & & & \\
\hline Burn &,- 065 & ,027 &,- 256 &,- 114 &,- 134 & & & & & & \\
\hline Agot &,$- 361 \dagger$ &,- 045 &,$- 506 \dagger$ &,$- 555^{*}$ &,- 347 & ,338 & & & & & \\
\hline Desp &,- 282 &,- 079 &,- 235 &,$- 518 *$ &,- 197 & 177 &, $526 \dagger$ & & & & \\
\hline B real &,- 110 &,- 202 & 092 &,- 163 & ,219 & ,078 &,- 292 &,- 068 & & & \\
\hline Activ &,- 011 &,- 021 &, 074 & ,000 &,$- 372 \dagger$ & ,049 & ,242 & ,203 &,- 217 & & \\
\hline Evit &,- 039 & ,288 &,- 215 & ,062 &,- 246 & 296 & 291 &,- 005 &,$- 441 \dagger$ & 283 & \\
\hline Pasiv & ,268 &, $367 \dagger$ &,- 114 & , 156 &,- 122 & 071 & 009 &,- 147 &,- 248 & ,221 & ,719* \\
\hline
\end{tabular}

$* \mathrm{p}<.000, * * \mathrm{p}<.001, \dagger \mathrm{p}<.005$

A continuación se someten a una breve discusión, los datos encontrados en la muestra de trabajadores varones y mujeres de la empresa que ha sido objeto de nuestro estudio.

\section{DISCUSIÓN}

Las empresas están experimentando diversos cambios motivados por la alta tecnologización, los mercados globales y las nuevas estructuras administrativas, que tienden hacia la horizontalidad dentro de las organizaciones (Robbins, 1999). Hoy se necesitan, por tanto, trabajadores con habilidades diferentes. Dentro de las habilidades personales se requiere el orden, la resiliencia, la capacidad de negociación y de toma de decisiones, la capacidad de automotivarse, el pensamiento estratégico y el liderazgo. En cuanto a las competencias sociales se relievan el manejo de conflictos, el trabajo en equipo, la empatía, la inteligencia emocional y los valores (Zavala, 2009).

Asimismo, con un enfoque orientado hacia el bienestar, la psicología positiva pone énfasis en la organización de empresas saludables que promuevan la calidad de vida en el trabajo, que se hace evidente a 
través de salarios justos y adecuados, apoyos sociales, entornos seguros y saludables, formas de negociación colectiva e individual libre, crecimiento y desarrollo organizacional y personal, integración y aceptación social, participación en la toma de decisiones y formas democráticas de poder (García, 2007); que devienen en características de puestos de trabajo saludables. En ese sentido, como indican Salanova et al (2005), las características de un puesto saludable, dentro del marco de la psicología positiva, son el control del puesto, la oportunidad del uso de habilidades, la variedad, la claridad en las tareas, las oportunidades de contacto social y la retroalimentación en el trabajo.

Estas características tornan los centros de trabajo, en lugares cómodos, seguros y felices; de modo que se favorece la salud mental de los trabajadores, lo cual implica un estado emocional positivo y un modo de pensar compasivo consigo mismo y con los demás, que tiene alcance futuro a través de expectativas positivas y sentimientos de esperanza (Vázquez \& Hervás, 2008). Todo ello, repercute también en menores índices de estrés laboral, pero como señalan Guillén et al (2000), conviene no olvidar que el estrés no se origina siempre en factores externos o en factores organizacionales, sino que puede desencadenarse por las características personales del trabajador, es decir, por su vulnerabilidad ante determinados estímulos. Es así que, los estilos de afrontamiento, pueden mediar entre los niveles de estrés percibido y la satisfacción con el trabajo, expresada en términos de felicidad.

Es en este contexto que se desarrolló la presente investigación, con el objeto de encontrar relaciones significativas entre la felicidad, el estrés crónico en el trabajo (o síndrome de "burnout") y los estilos de afrontamiento; dentro de una empresa privada de la ciudad de Arequipa. Los resultados indican, que entre los trabajadores evaluados, existen altos niveles de felicidad, y niveles bajos y moderados de estrés crónico. Aunque parece ser que los varones experimentan menores niveles de burnout $(79,5 \%$ de nivel leve) que las mujeres (63,3\% de burnout leve). Sin embargo, las relaciones negativas entre felicidad y estrés crónico son más fuertes entre las mujeres, de modo que las mujeres más felices son las que experimentan menos agotamiento emocional, y las que se sienten más realizadas personalmente, y están satisfechas con la vida tienen menos agotamiento emocional y despersonalización. En los varones, los que tienen mayor alegría de vivir, se sienten más realizados personalmente, también se sienten menos despersonalizados. Asimismo, entre los varones, quienes tienen mayor edad presentan niveles menores de agotamiento emocional y despersonalización. Dado que los niveles de felicidad son elevados y las relaciones con el síndrome de burnout son inversas, es posible como indican Rice, Lockenhoff y Carstensen (2002), que los estereotipos positivos de las personas mayores les permita mejorar sus habilidades, mientras que los estereotipos negativos disminuyan su autoeficacia. Además, entre los varones se encontraron relaciones negativas y significativas entre el estilo activo de afrontamiento y el número de hijos, mientras que las relaciones son positivas entre esta variable y el estilo pasivo, lo cual podría interpretarse de manera que mientras los varones tienen más hijos, asumen menos riesgos, ya que los estilos activos de afrontamiento se han relacionado con cardiopatía coronaria e hipertensión (Sandín, 1995).

Para el caso de las mujeres, el grado de instrucción se relaciona negativamente con la baja realización personal, lo cual es coherente con el hecho de que las mujeres que trabajan se sienten más satisfechas que los varones (Schultz, 1998), debido a que el trabajo para la mujer es percibido como una "conquista mayor", de lo que es percibido para el varón.

En cuanto a los estilos de afrontamiento, la mayoría de varones tiene un estilo de afrontamiento activo $(54,5 \%)$ en comparación con las mujeres $(36,7 \%)$, entre quienes predominan los estilos de afrontamiento evitativo y pasivo $(63,3 \%)$ más que en los varones $(45,5 \%)$. Precisamente, entre las mujeres, existe una relación negativa $(\mathrm{r}=-, 372)$ entre la alegría de vivir y el estilo activo de afrontamiento, lo cual puede sugerir que este estilo es más predominante entre quienes se sienten menos alegres, lo que a su vez se explica por la tendencia masculina hacia formas más agresivas de conducta (Gallardo, Forero, Maydeu \& Andrés, 2009), personalidades coléricas (Padilla, Peña \& Arriaga, 2006), patrón de conducta tipo A (García \& Berrios, 1999) y reactividad cardiovascular (Van Egeren \& Sparrow, 1989). También se ha encontrado que en los varones, hay relaciones positivas entre el 
estilo de afrontamiento pasivo y la despersonalización y el síndrome de burnout, lo cual significa que las formas pasivas de afrontamiento conducen a una vivencia mayor de estrés en los varones, así como a una conducta más irritable, negativa y distante. Esto también es consistente con el hecho de que las mujeres prefieren estilos de afrontamiento pasivo y que los varones que optan por estos estilos sienten malestar subjetivo, posiblemente porque contradicen los estereotipos sociales.

Por otro lado, dado que el estilo activo de afrontamiento correlaciona negativa $y$ significativamente con el estilo evitativo y el estilo pasivo, parece ser que tanto el estilo evitativo como el pasivo resultan similares $(r=, 719)$ y se diferencias significativamente de los estilos de afrontamiento activos. Los cual también aporta evidencias de que los varones utilizan estilos activos mientras que las mujeres utilizan los de tipo pasivo, ya que mientras los varones de nuestra muestra que utilizan el estilo pasivo tienen mayores niveles de despersonalización, las mujeres que utilizan los estilos pasivos tienen mayor sentido positivo de la vida.

Ahora bien, aunque se ha señalado que las personas optimistas prefieren estilos de afrontamiento activo (Salgado, 2009), no podemos afirmar que un estilo de afrontamiento determinado (sea activo, evitativo o pasivo), contribuya a la felicidad, ya que en general hemos encontrado relaciones significativas entre el síndrome de burnout y la felicidad, y el síndrome de burnout y el afrontamiento, mas no entre la felicidad y los estilos de afrontamiento. Posiblemente, un factor que influye en estas relaciones, es la tipología de estilos de afrontamiento que se ha empleado en este estudio, que se basa en el modelo de Magnuson (Labrador, 1996). Es probable que el modelo de Lazarus y Folkman (citados por Sandín, 1995) que diferencia entre los estilos de afrontamiento de confrontación, distanciamiento, autocontrol, búsqueda de apoyo social, aceptación de la responsabilidad, huida y evitación, planificación y reevaluación cognitiva; sea más apropiado para establecer relaciones con la felicidad y sus factores componentes.

Otra limitación de este estudio, radica en no haber considerado los puestos de los trabajadores, ya que numerosos autores señalan que los cargos de diferentes jerarquías tienen injerencia en el estrés laboral (García, 2003; Hernández, Almirall \& Franco, 2005; López, Morales, Murillo \& Rodríguez, 2006). En estudios posteriores, vamos a profundizar en este tipo de relaciones. Una tercera limitación se relaciona con la falta de equivalencia entre los géneros, por tal motivo es que se muestran los resultados en función del género, para minimizar los efectos de esta distribución desigual entre varones y mujeres. Además, puesto que los datos provienen de una sola empresa privada, no es posible generalizar los resultados a otros entornos organizacionales. A pesar de estas falencias, la investigación contribuye con el conocimiento de los beneficios de los enfoques psicológicos positivos en los ambientes laborales, ya que en nuestro medio son todavía escasos los estudios que se han realizado desde un enfoque de la psicología positiva en el trabajo.

Finalmente, aunque las medidas de estrés crónico no son elevadas, y son pocos los trabajadores que tienen niveles severos de burnout, se recomienda utilizar técnicas de relajación, meditación, biofeedback y de autocontrol, así como la práctica de ejercicio físico y la promoción de hábitos saludables en el trabajo, para disminuir los efectos del estrés laboral (Guillén et al, 2000). También sería importante emplear técnicas de programación neurolingüística, ya que han resultado eficientes para mejorar la comunicación organizacional (Marrero, Marrero \& Oramas, 2005), así como estrategias de conducta organizacional positiva y programas para mejorar el optimismo y la felicidad, como el propuesto por Fischman (2011). Todo ello, no sólo para promover la salud, sino también para generar una cultura organizacional positiva que coadyuve con el incremento de la productividad y la seguridad del entorno laboral.

Queremos también agradecer a los participantes de este estudio y a las autoridades de esta empresa privada, ya que sin su colaboración no hubiera sido posible su realización. 


\section{REFERENCIAS}

Alarcón, R. (2006). Desarrollo de una Escala Factorial para medir la Felicidad. Revista Interamericana de Psicología, 40(1), 99-106.

Alarcón, R. (2009). Psicología de la felicidad. Introducción a la psicología positiva. Lima: Universidad Ricardo Palma.

Alarcón, R. (2011). Ensayos sobre psicología contemporánea. Lima: Universidad Ricardo Palma.

Alonso, F. (2009). ¿Por qué trabajamos? El trabajo entre el estrés y la felicidad. España: Ediciones Díaz de Santos.

Apiquian, A. (2007). El síndrome de burnout en las empresas. Tercer Congreso de Escuelas de Psicología de las Universidades Red Anáhuac Mérdida, Yucatán. Recuperado de: www.ucm.es/ cont/descargas/documento33772.pdf

Ardila, R. (1991). Psicología del desempleado. Revista Latinoamericana de Psicología, 23(2), 207-227.

Boada, J.; de Diego, R. \& Agulló, E. (2004). El burnout y las manifestaciones psicosomáticas como consecuentes del clima organizacional y de la motivación. Psicothema, 16(1), 125-131.

Borda, M.; Navarro, E.; Aun, E.; Berdejo, H.; Racedo, K. \& Ruíz, J. (2007). Síndrome de burnout en estudiantes de internado del Hospital Universidad del Norte. Revista Salud Uninorte, 23(1), 43-51.

Fernández, M. (2010). El estrés laboral en los peruanos: hallazgos recientes. Teoría e investigación en psicología, 19, 37-59.

Fischman, D. (2010). La alta rentabilidad de la felicidad. Lima: UPC.

Fredrickson, B. L. (2009). Vida positiva. Cómo superar las emociones negativas y prosperar. Bogotá: Norma.

Gallardo, D.; Forero, C. G.; Maydeu, A. \& Andrés, A. (2009). Desarrollo del comportamiento antisocial: Factores psicobiológicos, ambientales e interacciones genotipo-ambiente. Revista de Neurología, 48(4), 191-198.

García, A. (2003). Discriminación de factores de estrés laboral entre ejecutivos y personal operativo en una micro empresa del Valle de México. Recuperado de: www.colparmex.org/Revista/Art3/15.pdf

García, M. A. \& Berrios, M. P. (1999). El significado del trabajo en personas con patron de conducta tipo A. Psicothema, 11(2), 357-366.

Gil-Monte, P. R. \& Peiró, J. M. (1999a). Perspectivas teóricas y modelos interpretativos para el estudio del síndrome de quemarse por el trabajo. Anales de Psicología, 15(2), 261-268.

Gil-Monte, P. R. y Peiró, J. M. (1999b). Validez factorial del Maslach Burnout Inventory en una muestra multiocupacional. Psicothema, 11(3), 679-689.

Goleman. D. (2010). Inteligencia emocional en la empresa. Buenos Aires: Vergara.

Gómez, V. (2010). Assesment of psychosocial stressors at work: Psychometric properties of the Spanish version of the ERI (Effort-Reward Imbalance) Questionnaire in Colombian workers. Revista de Psicología del Trabajo y de las Organizaciones, 26(2), 147-156.

Greco, C. (2010). Las emociones positivas: $\mathrm{Su}$ importancia en el marco de la promoción de la salud mental en la infancia. Liberabit, 16(1), 81-94.

Guillén, C.; Guil, R, \& Mestre, J. M. (2000). Estrés laboral. En Guillén, C. y Guill, R. (comps.), Psicología del trabajo para relaciones laborales (p. 268-296). Madrid: McGraw-Hill.

Hernández, J. S.; Almirall, P. J. \& Franco, J. G. (2005). Procedimiento para la verificación, diagnóstico y vigilancia de la salud laboral en la empresa: CBS. Estudio piloto. Revista Cubana de Salud y Trabajo, 6(1), 36-41.

Izquierdo, T. \& Alonso, H. J. (2010). Valores culturales y consecuencias psicosociales del desempleo en América Latina. Revista de Psicología del Trabajo y de las Organizaciones, 26(2), 123-133.

Jáuregui, E. (2008). Tomarse el humor en serio: Aplicaciones positivas de la risa y el humor. En Vázquez, C. y Hervás, G. (eds.) Psicología Positiva Aplicada, (pp. 283-309). España: Editorial Desclée de Brouwer.

Labrador, J. F. (1996). El estrés. Nuevas técnicas para su control. España: Grupo Correo de Comunicaciones.

Lamas, H. (2004). Promoción de la salud: Una propuesta desde la psicología positiva. Liberabit, 10(1), 45-67.

López, B. \& Osca, A. (2010). Influencia de algunas variables organizacionales sobre la salud y la accidentabilidad laboral. Anales de Psicología, 26(1), 89-94. 
López, L. (1993). Evaluación conductual en la empresa. Revista Latinoamericana de Psicología, 25(3), 375-402.

López, R.; Morales, P.; Murillo, M. \& Rodríguez, L. (2006). Relación entre la conducta tipo A y el nivel jerárquico en las organizaciones. (Trabajo de investigación no publicado).

Marrero, V.; Marrero, M. L. \& Oramas, A. (2005). La programación neurolingüística (PNL) como método de intervención psicológica para el mejoramiento de la comunicación organizacional. Revista Cubana de Salud y Trabajo, 40(2), 41-47.

Moreno, M. P.; Ríos, M. L.; Canto, J.; San Martín, J. \& Perles, F. (2010). Satisfacción laboral y burnout en trabajos poco cualificados: Diferencias entre sexos en población inmigrante. Revista de Psicología del Trabajo y de las Organizaciones, 26(3), 255-265.

Niven, D. (2011). Los 100 secretos de la gente feliz. Colombia: Norma.

Ospina, D. E. (2007). Medición de la resiliencia. Investigación y Educación en Enfermería, 25(1), 58-65.

Padilla, V. M.; Peña, J. A. \& Arriaga, A. E. (2006). Patrones de personalidad tipo A o B, estrés laboral y correlates psicofisiológicos. Psicología y Salud, 16(1), 79-85.

Page, N.; Govindji, R.; Carter, D. \& Linley, P. A. (2008). Gestión positiva de los recursos humanos: Aplicaciones de la psicología positiva a lo largo del ciclo vital del trabajador. En Vázquez, C. y Hervás, G. (eds.) Psicología Positiva Aplicada, (pp. 429458). España: Editorial Desclée de Brouwer.

Pérez, A. M. (1996). Los significados sociales en torno al trabajo. Revista Latinoamericana de Psicología, 28(1), 13-30.

Rice, C. J.; Lockenhoff, C. E. \& Carstensen, L. L. (2002). En busca de independencia y productividad: cómo influyen las culturas occidentales en las explicaciones individuales y científicas del envejecimiento. Revista Latinoamericana de Psicología, 34(1-2), 133-154.

Robbins. S. (1999). Comportamiento organizacional. México: Prentice Hall.

Fecha de recepción: 28 de abril del 2014

Fecha de aceptación: 28 de mayo del 2014
Roca, M. A. y Torres, O. (2001). Un estudio del síndrome de burnout y su relación con el sentido de coherencia. Revista Cubana de Psicología, 18(2), 120-126.

Salanova, M. (2008). Organizaciones saludables: Una aproximación desde la psicología positiva. En Vázquez, C. y Hervás, G. (eds.) Psicología Positiva Aplicada, (pp. 403-427). España: Editorial Desclée de Brouwer.

Salanova, M.; Martínez, I. M. \& Llorens, S. (2005). Psicología organizacional positiva. En Palací, F. J. (coord.) Psicología de la Organización, (pp. 349376). Madrid: Pearson Education.

Salgado, A. C. (2009). Felicidad, resiliencia y optimismo en estudiantes de colegios nacionales de Lima. Liberabit, 15(2), 133-141.

Sandín, B. (1995). El estrés. En Belloch, A.; Sandín, B. y Ramos, F. (comps.). Manual de psicopatología II (p.: 3-52). Madrid: McGraw-Hill.

Schultz, D. P. (1998). Psicología industrial. 3ra edición. Colombia: McGraw-Hill.

Seligman, M. (2006). La auténtica felicidad. Buenos Aires: Vergara.

Van Egeren, L. F. \& Sparrow, A. W. (1989). Laboratory stress testing to assess real-life cardiovascular reactivity. Psychosomathic medicine, 51, 1-9.

Vázquez, C. \& Hervás, G. (2008). Salud positiva: Del síntoma al bienestar. En Vázquez, C. y Hervás, G. (eds.) Psicología Positiva Aplicada, (pp. 17-39). España: Editorial Desclée de Brouwer.

Villanueva, V. (2004). Psicología positiva, zona de flujo positivo, seguridad y compañía. Liberabit, 10(1), 89-95.

Waugh, C. E. \& Fredrickson, B. L. (2006). Nice to know you: Positive emotions, self, other overlap, and complex understanding in the formation of a new relationship. The Journal of Positive Psychology, 1(2), 93-106.

Zavaleta, R. (2009). Aprenda a ser feliz. Lima: Planeta. 